\title{
Tópicos de Probabilidade através da Resolução de Problemas
}

Probability topics through Problem Solving

\author{
Alessandra Negrini Dalla Barba ${ }^{1}$ \\ Bruno Rodrigo Teixeira²
}

\section{Resumo}

Este artigo tem por objetivo apresentar uma proposta para o ensino de alguns tópicos do conteúdo Probabilidade por meio da Resolução de Problemas, voltada para o trabalho com alunos de Ensino Médio. A perspectiva da Resolução de Problemas adotada é como ponto de partida para o ensino de conceitos matemáticos, conforme apresentado por Onuchic e Allevato (2009), e, por isso, são destacadas algumas ações e sugestões para o desenvolvimento de uma prática em sala de aula nessa perspectiva. Na proposta são apresentados os enunciados de problemas escolhidos, de acordo com os conceitos a serem introduzidos, e para cada um deles são indicadas possíveis resoluções e encaminhamentos que podem ser adotados pelo professor a partir de resoluções apresentadas com vistas à formalização dos conceitos. Entre as expectativas com a aplicação desta proposta podem ser destacadas a introdução de conceitos com compreensão e, além disso, maior participação dos alunos nas tarefas propostas e envolvimento com a sua aprendizagem.

Palavras-chave: Educação Matemática. Resolução de Problemas. Probabilidade.

\section{Abstract}

In this paper, we present a didactic proposal for teaching some Probability topics through Problem Solving in High School Mathematics classes. In this Problem Solving perspective, according to Onuchic and Allevato (2009), a problem is starting point for teaching mathematical concepts and taking this into account, we stand out some actions and suggestions for the development of a classroom practice involving this approach. In the proposal, we present problem statements, according to the concepts to be introduced, and for each of them are described possible resolutions and suggestions for teachers to formalize the concepts. The expectations for the use of this proposal in the classroom include introducing a new concept with comprehension, increasing students' participation in the tasks and engaging them with their own learning.

Keywords: Mathematics Education. Problem Solving. Probability.

\footnotetext{
${ }^{1}$ Mestra em Matemática Aplicada e Computacional. Docente do Departamento de Matemática da Universidade Pitágoras Unopar. E-mail: alessandra.barba@kroton.com.br

2 Doutor em Ensino de Ciências e Educação Matemática. Docente do Departamento de Matemática e do Programa de Pós-Graduação em Ensino de Ciências e Educação Matemática da Universidade Estadual de Londrina (UEL). E-mail: bruno@uel.br
} 


\section{Introdução}

De acordo com as Orientações Curriculares para o Ensino Médio, o estudo de Probabilidade tem sido recomendado em todas as etapas da Educação Básica, principalmente no Ensino Médio, devido à "importância das idéias de incerteza e de probabilidade, associadas aos chamados fenômenos aleatórios, presentes de forma essencial nos mundos natural e social." (BRASIL, 2006, p. 78).

Com relação ao ensino de Probabilidade, assim como dos demais conteúdos matemáticos da Educação Básica, Rotunno (2007, p.31) destaca que propostas curriculares do início da década de 1990 "consideravam como ponto de partida [...] a definição do conceito, a formalização. Os conceitos matemáticos eram vistos como bens culturais transmitidos hereditariamente como um produto final e acabado." Assim, o enfoque era a apresentação das definições e aplicação das mesmas em exercícios para fixação do conteúdo.

No entanto, a partir de 1998, documentos oficiais como os PCN (BRASIL, 1998), PCN+ (BRASIL, 2002), Orientações Curriculares para o Ensino Médio (BRASIL, 2006) começaram a sugerir que o estudo de conteúdos matemáticos pudesse ser feito, por exemplo, através da resolução de problemas tendo em conta suas potencialidades para a aprendizagem dos alunos, potencialidades essas que têm sido destacadas em diversos estudos (STEIN; BOALER; SILVER, 2003; VAN DE WALLE, 2009; ONUCHIC; ALLEVATO, 2009; CAI, 2010; ONUCHIC; ALLEVATO, 2011).

Diante dessas potencialidades e de experiências anteriores vivenciadas pelos autores deste artigo em que a Resolução de Problemas foi utilizada como ponto de partida para a introdução de diferentes conteúdos matemáticos, e dos estudos realizados pela primeira autora na disciplina "Contagem", ministrada pelo segundo autor em um curso de Especialização em Educação Matemática - em que eram discutidos diferentes aspectos sobre Análise Combinatória e Probabilidade e a respeito do ensino desses conteúdos - surgiu a intenção de 
desenvolver uma monografia ${ }^{3}$ para a conclusão do curso que apresentasse uma proposta de ensino de Probabilidade nessa perspectiva, a qual originou o presente trabalho.

Tendo em vista estas considerações, este artigo tem por objetivo apresentar uma proposta para o ensino de alguns tópicos de Probabilidade, através da Resolução de Problemas, voltada para o trabalho com alunos de Ensino Médio.

Com base neste objetivo, a seguir são apresentados alguns aspectos teóricos relacionados ao ensino de Probabilidade a partir da Resolução de Problemas, sugestões metodológicas que podem subsidiar o trabalho do professor em sala de aula, e, por fim, o enunciado de cada problema relacionado ao tema, acompanhado de possíveis resoluções e sugestões para a formalização dos conteúdos.

Por fim, são apresentadas algumas considerações destacando expectativas para a aplicação desta proposta para alunos de Ensino Médio, além de potencialidades de sua aplicação na formação inicial e continuada de professores de Matemática.

\section{Ensino de Probabilidade a partir da Resolução de Problemas: algumas considerações}

O ensino de Probabilidade partindo da Resolução de Problemas com alunos de Ensino Médio têm sido discutido em alguns trabalhos como Lopes (2011) e Marocci e Nacarato (2013).

Lopes (2011) apresenta os resultados da aplicação de uma proposta envolvendo Probabilidade, jogos e Resolução de Problemas. O objetivo do autor era observar as percepções de três professoras de Ensino Médio, sobre suas práticas de ensino, a partir da aplicação da proposta.

A pesquisa foi desenvolvida ao longo de dois anos com turmas do $2^{\circ}$ ano do Ensino Médio. Uma das etapas desta foi a aplicação da proposta pelas ${ }^{3}$ Barba (2015). 
professoras, após discussão do material entre o pesquisador - 0 autor - e as mesmas. As docentes perceberam que houve melhora no desempenho dos alunos no estudo de Probabilidade com base nessa proposta em comparação com o estudo a partir das aulas tradicionais. De acordo com o autor, o "uso da Resolução de Problemas acarreta mais trabalho ao professor, mas se adequadamente utilizada pode contribuir significativamente com a melhoria do processo de ensino e aprendizagem" (LOPES, 2011, p.11).

Marocci e Nacarato (2013) apresentam parte de uma pesquisa de mestrado envolvendo Probabilidade e Resolução de Problemas, "cujo tema central é o movimento das significações probabilísticas" (p.102) entre alunos do $1^{\circ}$ ano do Ensino Médio, sendo os trabalhos desenvolvidos em parceria com a professora da turma. Na situação relatada o objetivo era promover a discussão com os alunos sobre as ideias de eventos certos, impossíveis e possíveis a partir de situações cotidianas.

Apesar de a tarefa apresentada não constituir "um problema tão especial" (MAROCCI; NACARATO, 2013, p.119), as discussões observadas foram muito ricas, com grande participação dos alunos. Em relação à Resolução de Problemas, as autoras afirmam que o estudo da Probabilidade a partir desta metodologia auxilia na aproximação dos conceitos com situações cotidianas, favorecendo o estabelecimento de relações e a significação de palavras como "incerto", "certo", "possível", "impossível", "sorte", entre outras, além da ampliação da capacidade dos alunos em resolver problemas.

Com a análise de trabalhos como esses supracitados é possível observar contribuições do uso da Resolução de Problemas para os processos de ensino e de aprendizagem de conceitos relacionados à Probabilidade.

Na próxima seção são apresentados aspectos metodológicos relacionados à proposta elaborada, os quais podem subsidiar o trabalho do professor em sala de aula.

\section{Sugestão para o trabalho com a proposta de ensino}


Neste trabalho é apresentada uma proposta de ensino, envolvendo o conteúdo matemático Probabilidade através da Resolução de Problemas, destinada a alunos de Ensino Médio. Entre os conceitos relativos à Probabilidade, foram selecionados: as definições de espaço amostral e de evento, o cálculo de probabilidades, probabilidade da união de dois eventos e probabilidade condicional ${ }^{4}$.

Considerando que a perspectiva da Resolução de Problemas adotada é como ponto de partida para o ensino de Matemática, nessa seção são apresentados alguns aspectos relacionados à aplicação desta metodologia ${ }^{5} \mathrm{em}$ sala de aula. Nessa perspectiva de ensino, professor e alunos "juntos desenvolvem esse trabalho, e a aprendizagem realiza-se de modo cooperativo e colaborativo em sala de aula" (ONUCHIC; ALLEVATO, 2009, p.97).

A organização de uma aula segundo a Resolução de Problemas pode ser feita a partir das seguintes ações, com base em etapas apresentadas por Onuchic e Allevato (2009):

- seleção do problema envolvendo preferencialmente conteúdos ainda não trabalhados em sala de aula (neste caso, os problemas já foram selecionados);

- leitura individual e em grupos do enunciado do problema, por parte dos alunos, com o auxílio do professor quando necessário;

- resolução do problema, sendo o professor um orientador e incentivador do trabalho desenvolvido pelos alunos;

- discussão das resoluções propostas pelos alunos e apresentadas para toda a turma, de modo que os mesmos defendam suas ideias e esclareçam suas dúvidas, buscando um consenso sobre o resultado correto para o problema em estudo;

\footnotetext{
${ }^{4} \mathrm{Na}$ monografia são apresentados outros, mas devido à limitação de páginas iremos nos restringir aos conceitos destacados.

${ }^{5}$ Este termo tem sido empregado na literatura para designar uma concepção de Resolução de Problemas em que "um problema é ponto de partida e orientação para a aprendizagem, e a construção do conhecimento far-se-á através de sua resolução" (ONUCHIC; ALLEVATO, 2009, p. 97).
} 
- formalização do conteúdo matemático pretendido pelo professor a partir de resoluções propostas.

Durante o desenvolvimento dessas ações "o professor terá que enfrentar situações inesperadas em sala de aula e, em algumas oportunidades, deverá alterar aquilo que tinha planejado, ainda mais, terá que estar atento às dificuldades apresentadas pelos alunos" (RODRIGUES, 1992, p. 29). Nesta perspectiva de trabalho (ONUCHIC; ALLEVATO, 2009), o professor ${ }^{6}$ precisa assumir um papel de orientador, auxiliando os alunos em suas dificuldades, mas permitindo que os mesmos encontrem a solução para os problemas de forma autônoma e participem ativamente na construção dos conceitos.

Para a proposta de ensino em tela foram selecionados enunciados de problemas aplicados em provas de vestibulares da Universidade Estadual de Londrina (UEL) ou no Exame Nacional do Ensino Médio (ENEM), sendo alguns adaptados em decorrência dos objetivos estabelecidos. Vale ressaltar que, caso o professor não apresente afinidade em relação aos enunciados, a partir da análise de como o trabalho foi planejado para esses problemas pode realizar um planejamento similar para outros ou adaptá-los à sua realidade. Além disso, pode destacar em seu planejamento outras possíveis resoluções e intervenções a serem realizadas no trabalho com os alunos.

Os problemas aqui empregados foram selecionados de acordo com a ordem em que os conceitos, em geral, são apresentados em livros didáticos adotados em escolas brasileiras (IEZZI et al., 2010; DANTE, 2013; SOUZA, 2013). Além disso, na proposta aqui apresentada, o foco está mais em evidenciar possibilidades para o professor a respeito de como partir da resolução e chegar à formalização, do que propriamente descrever os conceitos tais como aparecem já sistematizados nos materiais, pois as definições a serem apresentadas para os alunos na formalização do conteúdo podem ser selecionadas pelo professor, com

\footnotetext{
6 Para sugestões mais detalhadas, tanto no que se refere a que ações o professor pode desenvolver durante 0 trabalho com os alunos quanto no que diz respeito a como pode desenvolvê-las, sugerimos a leitura do trabalho de Teixeira e Santos (2017).
} 
os devidos cuidados, a partir dos livros didáticos mencionados e/ou de outros materiais utilizados em escolas de Ensino Médio.

Por meio do desenvolvimento desse trabalho, o objetivo é que os alunos compreendam os conceitos envolvidos antes de serem apresentadas as fórmulas, que são obtidas a partir de resoluções para os problemas e podem ser utilizadas posteriormente para a resolução de outras questões envolvendo o conteúdo. Por isso, é importante que nesse processo, eles percebam que as fórmulas podem ser úteis para a simplificação de cálculos e obtenção de soluções.

Na próxima seção é apresentada a proposta propriamente dita, indicando, para cada problema, o enunciado, possíveis resoluções e uma sugestão para a formalização do conteúdo a partir de algumas resoluções.

\section{A Introdução de Conteúdos através da Resolução de Problemas}

Nesta seção são apresentados os enunciados de problemas que podem ser utilizados para a introdução de alguns dos conceitos relacionados à Probabilidade estudados no Ensino Médio. A cada problema são destacadas sugestões de resoluções bem como uma possibilidade para a formalização do conteúdo a partir de determinada(s) resolução(ões).

Uma limitação dessa proposta consiste no fato de não serem apresentadas antecipações em relação a uma maior diversidade de resoluções que podem surgir no trabalho dos alunos com os problemas, possíveis equívocos dos alunos, assim como uma maior diversidade de propostas de exploração de erros e intervenções que podem ser realizadas pelo professor para auxiliá-los. Por ser um trabalho a ser executado pelos alunos, os mesmos podem empregar diferentes estratégias, ocasionando em diferentes resoluções, ainda que incompletas, para os problemas. Tendo isso em vista, recomendamos que, antes de aplicar essa proposta a seus alunos, o professor se atente a isso em seu planejamento e faça uma complementação com base em sua experiência em sala de aula, considerando as características da turma para a qual a mesma será direcionada, 
ou em discussões com seus pares. Exemplos de possíveis resoluções incorretas e possibilidade de exploração/intervenção por parte do professor foram colocados em forma de observação na possível resolução 3 para o problema 1 e na possível resolução 2 para o problema 3 .

O Quadro 1 apresenta quais conceitos podem ser formalizados a partir de cada problema.

Quadro 1: Organização dos conceitos abordados na proposta

\begin{tabular}{|c|c|}
\hline Problema & Conceito a ser formalizado \\
\hline 1 & $\begin{array}{r}\text { Definição de espaço amostral e de evento, cálculo da } \\
\text { probabilidade da ocorrência de um evento }\end{array}$ \\
\hline 2 & Probabilidade da união de dois eventos \\
\hline 3 & Probabilidade condicional \\
\hline
\end{tabular}

Fonte: os autores.

Os problemas 2 e 3, além de apresentarem os objetivos de acordo com o Quadro 1, também podem ser utilizados para a aplicação do cálculo de probabilidade já sistematizado no problema 1 como forma de evidenciar a compreensão dos alunos a esse respeito.

Quadro 2: Enunciado ${ }^{7}$ do Problema 1

As 23 ex-alunas de uma turma que completou o Ensino Médio há 10 anos se encontraram em uma reunião comemorativa. Várias delas haviam se casado e tido filhos. A distribuição das mulheres, de acordo com a quantidade de filhos, é mostrada no gráfico abaixo.

\footnotetext{
${ }^{7}$ Adaptado da prova do ENEM de 2005. Disponível em:<http://download.inep.gov.br/educacao_basica/enem/provas/2005/2005_amarela.pdf>.Acesso em 28 nov. 2017.
} 


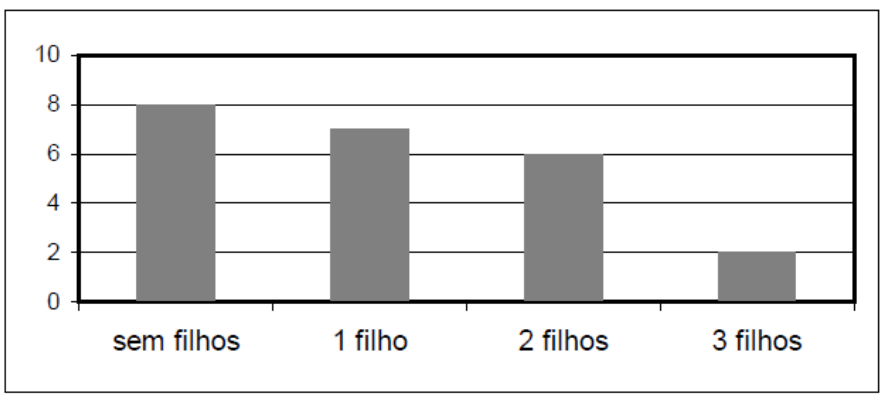

Um prêmio foi sorteado entre todos os filhos dessas ex-alunas. Qual a chance de que a criança premiada seja um(a) filho(a) único(a)?

Fonte: Adaptado de INEP (2005).

Possíveis resoluções:

1) A situação pode ser representada, a partir das informações indicadas no gráfico, da seguinte forma

8 ex-alunas não possuem filhos

7 ex-alunas possuem exatamente 1 filho(a) cada

6 ex-alunas possuem exatamente 2 filhos(as) cada

2 ex-alunas possuem exatamente 3 filhos(as) cada.

Sendo assim, o total $(T)$ de filhos de todas as mulheres que se encontraram na reunião é dado por

$$
T=8 \cdot 0+7 \cdot 1+6 \cdot 2+2 \cdot 3=0+7+12+6=25 .
$$

Como existem 7 crianças que são filhos(as) únicos(as), então a chance de a criança sorteada ser um(a) filho(a) único(a) é 7 em 25.

2) Pelo gráfico é possível observar que existem 25 crianças, filhos destas exalunas. Como todas as crianças possuem a mesma chance de serem sorteadas, então a chance de a criança sorteada ser um(a) filho(a) único(a) é de 7 dentre 25. 3) Como são 7 filhos únicos, a chance é de 1 em 7.

Observação: No caso de uma resolução desse tipo, o professor pode lançar mão de questionamentos, como os seguintes, com a intenção de auxiliar os alunos na percepção do equívoco:

- "Com a realização do sorteio, o que esse número 1 que você destaca representaria?" 
- "Ao ser realizado o sorteio de uma criança, pensando nos filhos únicos, que resultados diferentes poderiam ser obtidos?"

- "Ao ser realizado o sorteio de uma criança, pensando agora no total de crianças, que resultados diferentes poderiam ser obtidos?"

\section{Proposta para a formalização do conteúdo:}

Nesta situação o objetivo é sortear uma criança dentre um total e verificar a chance dela ser um filho único. Após as apresentações dos alunos e um consenso sobre a resolução correta, o professor pode discutir com os alunos que por se tratar de um sorteio, não é possível prever o resultado antes que o mesmo seja realizado, mesmo que este ocorra diversas vezes. Este tipo de fenômeno pode ser definido como um experimento aleatório.

Partindo do número 25 apresentado em resoluções como as possíveis resoluções 1 e 2, pode discutir o significado desse número na situação em questão e formalizar o conceito de espaço amostral. Nesse contexto são consideradas mulheres sem filhos, com filhos únicos, com exatamente dois filhos ou com exatamente três filhos, sendo um total de 25 crianças que podem ser ganhadoras do prêmio. Ao conjunto de todos os possíveis resultados damos o nome de espaço amostral.

Assim, no problema em estudo, o espaço amostral é dado pelo conjunto $(\Omega)$ de todas as crianças, que pode ser representado da seguinte forma

$$
\Omega=\left\{C_{1}, C_{2}, C_{3}, \ldots, C_{25}\right\}
$$

onde cada $C_{i}, 1 \leq i \leq 25$, representa uma das 25 crianças consideradas nesta situação.

Desta forma, nesse instante inicial, é importante apresentar as definições formais, com base em livros didáticos, por exemplo, para os conceitos de experimento aleatório e espaço amostral.

Partindo do número 7 apresentado em resoluções como as possíveis resoluções 1 e 2, o professor pode questionar os alunos de modo que evidenciem 
que se refere a quantidade de elementos de determinado conjunto, aquele formado pelos filhos únicos.

Além disso, pode destacar que a criança sorteada pode ser filho único, ter um único irmão ou ter exatamente dois irmãos. Assim, a partir do espaço amostral, podemos destacar os subconjuntos $F=\left\{C_{1}, C_{2}, C_{3}, \ldots, C_{7}\right\}$, $U=\left\{C_{8}, C_{9}, C_{10}, \ldots, C_{19}\right\}$ e $D=\left\{C_{20}, C_{21}, C_{22}, \ldots, C_{25}\right\}$ em que $F$ representa o conjunto dos filhos únicos, $U$ o conjunto das crianças com um único irmão e $D$ das crianças com exatamente dois irmãos. Cada um destes subconjuntos é considerado um evento ${ }^{8}$, conceito que também pode ser definido com base nas características do problema em estudo, conforme a explicação anterior.

Após definir os conceitos iniciais, com base nas definições presentes em livros didáticos e outros materiais, é possível introduzir o cálculo da probabilidade de ocorrer um evento a partir do questionamento apresentado no problema.

Como a situação envolve um total de 25 crianças, dentre as quais apenas 7 são filhos únicos, então o espaço amostral $\Omega$ é composto por 25 elementos e o conjunto $F$, definido anteriormente, por 7 elementos. Como todas as crianças possuem a mesma chance de serem sorteadas, por se tratar de um experimento aleatório, então o professor pode definir que a chance de a criança premiada ser um(a) filho(a) único(a) é determinada por meio da probabilidade, que é calculada como a razão entre a quantidade de crianças que são filhos únicos e a quantidade total de crianças, ou seja, a probabilidade de a criança sorteada ser filha única é de $\frac{7}{25}$.

Partindo dessas informações, se o espaço amostral $\Omega$ é tal que seu número de elementos é $n(\Omega)=25$, e se $F$ corresponde ao evento tal que

\footnotetext{
${ }^{8}$ As definições de evento certo e evento impossível também podem ser abordados aproveitando o contexto do problema, por exemplo, questionando os alunos como seria o conjunto das crianças que possuem no máximo três irmãos, o das crianças que possuem exatamente três irmãos, etc.
} 
$n(F)=7$, então a probabilidade de ocorrer o evento $F$, denotada por $P(F)$, será dada por $P(F)=\frac{n(F)}{n(\Omega)}=\frac{7}{25}$.

Neste momento, o professor pode apresentar a definição formal, conforme destacada nos livros didáticos e/ou em outros materiais empregados no estudo de Matemática no Ensino Médio.

Por fim, pode trabalhar com os alunos a representação decimal $(0,28)$ e percentual (28\%) do resultado obtido, tendo em vista que essas representações também são frequentemente utilizadas.

Quadro 3: Enunciado 9 do Problema 2

De um total de 500 estudantes da área de exatas, 200 estudam Cálculo Diferencial e 180 estudam Álgebra Linear. Esses dados incluem 130 estudantes que estudam ambas as disciplinas. Qual é a probabilidade de que um estudante escolhido aleatoriamente esteja estudando Cálculo Diferencial ou Álgebra Linear?

Fonte: Adaptado de UEL (2008).

\section{Possíveis resoluções:}

1) O seguinte diagrama pode ser construído, com $C$ representando o evento "estudar Cálculo Diferencial" e $A$ o evento "estudar Álgebra Linear".

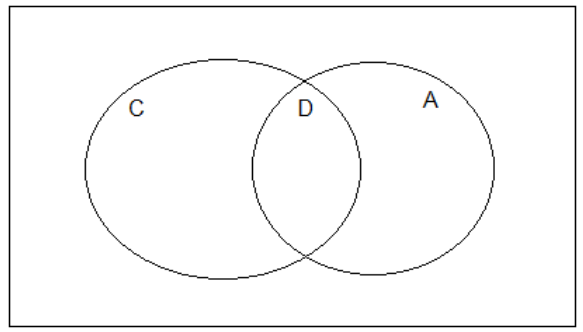

Como existem alunos que estudam ambas as disciplinas em questão, então existe uma interseção entre os conjuntos $C$ e $A$, a qual pode ser denotada por $D$ e correspondente ao evento "estudar Cálculo Diferencial e Álgebra Linear".

${ }^{9}$ Adaptado da Prova específica de Matemática do Vestibular da UEL de 2008. Disponível em: <http://www.cops.uel.br/vestibular/2008/provas/P10.PDF>. Acesso em 28 nov. 2017. 
O conjunto $D$ contém 130 estudantes, que são os que estudam ambas as disciplinas, assim $n(D)=130$. Como 200 estudantes compõem o conjunto $C$ então $n(C)=200$. Disto segue que

$$
n(C)-n(D)=200-130=70
$$

ou seja, 70 alunos estudam apenas Cálculo Diferencial. De modo análogo, como $A$ contém 180 estudantes, $n(A)=180$ e tem-se que

$$
n(A)-n(D)=180-130=50
$$

então 50 alunos estudam apenas Álgebra Linear.

Dentre os 500 alunos da área de exatas, a quantidade $(Q)$ de alunos que estudam Cálculo Diferencial ou Álgebra Linear é dada por

$$
Q=n(D)+(n(C)-n(D))+(n(A)-n(D))=130+70+50=250
$$

Portanto, a probabilidade de que um estudante escolhido aleatoriamente esteja estudando Cálculo Diferencial ou Álgebra Linear é $\frac{250}{500}$, ou ainda, $\frac{1}{2}=0,5=50 \%$.

2) Existem 180 estudantes de Álgebra Linear. Além disso, dos 200 estudantes de Cálculo Diferencial, 130 estudam também Álgebra Linear. Considerando $C$ como o evento "estudar Cálculo Diferencial", A sendo "estudar Álgebra Linear" e $D$ correspondente a "estudar Cálculo Diferencial e Álgebra Linear", e analisando também o diagrama a seguir, é possível concluir que

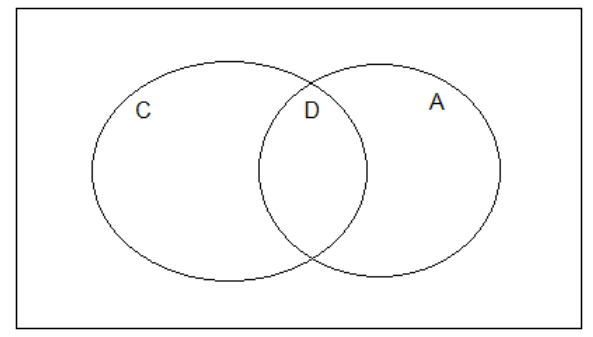

$n(C \cup A)=n(C)+n(A)-n(D)$

e como $D=C \cap A$,

$$
n(C \cup A)=n(C)+n(A)-n(C \cap A) .
$$

Logo, a quantidade de alunos que estudam Cálculo Diferencial ou Álgebra Linear é dada por 


$$
n(C \cup A)=180+200-130=250
$$

Portanto, a probabilidade de que um aluno, escolhido aleatoriamente, esteja estudando Cálculo Diferencial ou Álgebra Linear é $\frac{250}{500}=\frac{1}{2}$, ou ainda, $\frac{1}{2}=0,5=50 \%$

Proposta de formalização do conteúdo:

Neste problema o espaço amostral $\Omega$ é composto por 500 elementos, os quais correspondem ao total de alunos da área de exatas. São destacados dois eventos dentre este espaço amostral, a saber:

\section{Evento $C$ : "estudar Cálculo Diferencial" Evento $A$ : "estudar Álgebra Linear"}

Assim, tem-se

$$
n(\Omega)=500 ; n(C)=200 ; n(A)=180 \text {. }
$$

O evento $C \cup A$, definido por "estudar Cálculo Diferencial ou Álgebra Linear", envolve todos os alunos que estudam pelo menos uma destas disciplinas, ou seja, corresponde à união dos eventos $C$ e $A$. Assim, como indicado na resolução 1, podemos identificar as quantidades de alunos que estudam apenas uma das disciplinas, somá-los e adicionar o resultado ao total de alunos que estudam ambas as disciplinas para obter o total, que é igual a $n(C \cup A)=250$.

Partindo das possíveis resoluções, temos que

$$
P(C \cup A)=\frac{250}{500}
$$

Mas, $n(C \cup A)=250$ e $n(\Omega)=500$, assim

$$
P(C \cup A)=\frac{n(C \cup A)}{n(\Omega)}
$$

Desta forma, esta consiste em uma possibilidade para o cálculo da probabilidade da união de dois eventos.

Contudo, partindo da possível resolução 2, obtemos que 


$$
n(C \cup A)=180+200-130=250
$$

ou seja,

$$
n(C \cup A)=n(C)+n(A)-n(C \cap A)
$$

Sendo assim, a probabilidade de o evento $C \cup A$ ocorrer é dada por

$$
P(C \cup A)=\frac{n(C \cup A)}{n(\Omega)}=\frac{n(C)+n(A)-n(C \cap A)}{n(\Omega)}=\frac{n(C)}{n(\Omega)}+\frac{n(A)}{n(\Omega)}-\frac{n(C \cap A)}{n(\Omega)}
$$

Mas sabemos que

$$
P(C)=\frac{n(C)}{n(\Omega)}, P(A)=\frac{n(A)}{n(\Omega)} \text { e } P(C \cap A)=\frac{n(C \cap A)}{n(\Omega)}
$$

Portanto,

$$
P(C \cup A)=P(C)+P(A)-P(C \cap A)
$$

que corresponde a forma de se obter a probabilidade da união de dois eventos comumente apresentada em livros didáticos. Assim, após a obtenção desta expressão, o professor pode apresentar a definição formal da probabilidade da união de dois eventos, considerando todos os elementos indicados e empregando-a para quaisquer dois eventos de um espaço amostral, tomando por base os livros didáticos para o Ensino Médio que tratam sobre o assunto.

Quadro 4: Enunciado ${ }^{10}$ do Problema 3

No diagrama a seguir, o espaço amostral $S$ representa um grupo de amigos que farão uma viagem. O conjunto $A$ indica a quantidade de pessoas que já foram a Maceió e o conjunto $B$, a quantidade de pessoas que já foram a Fortaleza.

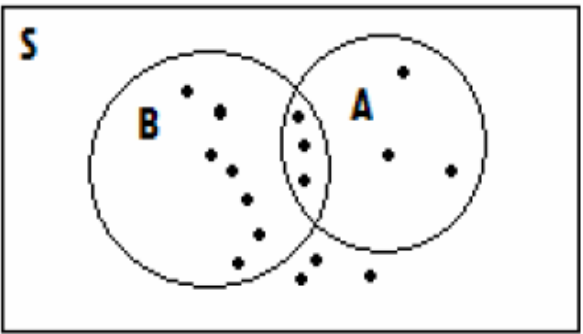

${ }^{10}$ Adaptado da Prova específica de Matemática do Vestibular da UEL de 2006. Disponível em: <http://www.cops.uel.br/vestibular/2006/provas/fisica_matematica.pdf >. Acesso em 28 nov. 2017. 
A empresa de turismo que está organizando a viagem fará o sorteio de uma passagem gratuita. Considerando que a pessoa sorteada já tenha ido para Fortaleza, determine a probabilidade de que ela também já tenha ido para Maceió.

Fonte: Adaptado de UEL (2006).

\section{Possíveis resoluções:}

1) Das 10 pessoas que já foram a Fortaleza, 3 também já foram a Maceió. Assim, a probabilidade $(P)$ de que a pessoa sorteada já tenha ido para Maceió, considerando que ela também já tenha ido para Fortaleza, é dada por

$$
P=\frac{3}{10}=0,3=30 \%
$$

2) Uma possível resolução apresentada pelos alunos pode conter apenas o cálculo da probabilidade de a pessoa ter ido a Maceió sem considerar a condição da mesma já ter ido a Fortaleza. Neste caso, dentre um total de 16 pessoas, 6 já foram a Maceió. Sendo assim, a probabilidade $P$ é dada por

$$
P=\frac{6}{16}=0,375=37,5 \%
$$

Observação: Para esta situação, o professor também pode questionar os alunos a respeito de alguns elementos do problema, conforme os exemplos apresentados a seguir, incentivando-os a refletirem sobre sua própria resposta, identificando as incoerências presentes:

- Todas as pessoas envolvidas na situação podem participar do sorteio? Como você chegou a essa conclusão?

- Todas as pessoas envolvidas na situação podem ser sorteadas? Como você obteve essa informação?

O professor pode apresentar os questionamentos diretamente ao grupo que apresentou a dificuldade ilustrada na resolução 2, durante o desenvolvimento das resoluções, ou no momento da plenária, no qual todos os grupos devem discutir a respeito das resoluções propostas, buscando identificar uma resposta correta ao problema. 


\section{Proposta de formalização do conteúdo:}

$\mathrm{Na}$ situação apresentada, o espaço amostral $S$ é composto por 16 elementos, que são as 16 pessoas envolvidas. $O$ evento $A$ pode ser definido a partir do conjunto $A$ como sendo "ter ido a Maceió" e o evento $B$, em função do conjunto $B$ apresentado, sendo definido como "ter ido a Fortaleza". A partir dos elementos indicados no diagrama tem-se

$$
n(S)=16 ; n(A)=6 ; n(B)=10 .
$$

Além disso, o evento "ter ido a Maceió e a Fortaleza" pode ser considerado, sendo definido por $A \cap B$, e é tal que

$$
n(A \cap B)=3
$$

O objetivo é a determinação da probabilidade de a pessoa sorteada pela empresa de turismo ter ido a Maceió, sabendo que ela já viajou para Fortaleza. Assim, neste sorteio não serão consideradas todas as pessoas no número total de resultados possíveis, conforme apresentado na resolução 2, mas apenas as que já foram a Fortaleza, pois esta foi a condição inicial imposta ao sorteado. Desta forma, haverá um novo espaço amostral a ser considerado que se restringirá a 10 pessoas, que correspondem as que já foram a Fortaleza.

Partindo da possível resolução 1, tem-se

$$
P=\frac{3}{10}=\frac{n(A \cap B)}{n(B)}
$$

Como esta probabilidade de ocorrer $A$ (ir a Maceió) está condicionada ao fato de que tenha ocorrido $B$ (ir a Fortaleza), vamos denominar esta probabilidade de condicional e denotá-la por $P(A / B)$.

Assim, segue que uma expressão que permite calcular essa probabilidade condicional, a probabilidade da ocorrência de um evento $A$ tendo ocorrido $B$, é a seguinte:

$$
P(A / B)=\frac{n(A \cap B)}{n(B)} .
$$

Observe que também podemos reescrever a expressão obtida em termos de probabilidades 


$$
P(A / B)=\frac{n(A \cap B)}{n(B)}=\frac{n(A \cap B)}{n(B)} \cdot \frac{n(S)}{n(S)}=\frac{n(A \cap B)}{n(S)} \cdot \frac{n(S)}{n(B)}=\frac{\frac{n(A \cap B)}{n(S)}}{\frac{n(B)}{n(S)}}=\frac{P(A \cap B)}{P(B)} .
$$

A definição formal de probabilidade condicional pode ser obtida nos livros didáticos de Matemática para o Ensino Médio e que tratam a respeito do tema, a qual pode ser apresentada aos alunos como forma de finalização para a formalização do conceito estudado.

\section{Considerações finais}

Neste trabalho foi apresentada uma proposta para o ensino de alguns tópicos do conteúdo Probabilidade através da Resolução de Problemas, considerando-a como ponto de partida para o ensino do conteúdo selecionado, de acordo com a perspectiva destacada por Onuchic e Allevato (2009). Para a elaboração da proposta foram selecionados problemas conforme os objetivos estabelecidos e os conteúdos a serem trabalhados, sendo a mesma direcionada a alunos de Ensino Médio por se tratar da etapa da Educação Básica na qual geralmente são introduzidos os conceitos de Probabilidade aqui abordados.

A Resolução de Problemas consiste em uma metodologia que pode contribuir com a introdução de conceitos ainda não estudados pelos alunos por possibilitar aos mesmos a compreensão e a construção de novos conceitos com base em seus conhecimentos prévios. Nota-se, ainda, que ela pode oportunizar uma maior participação dos alunos nas tarefas propostas e maior envolvimento com a sua aprendizagem, pois professor e alunos "juntos desenvolvem esse trabalho, e a aprendizagem realiza-se de modo cooperativo e colaborativo em sala de aula" (ONUCHIC; ALLEVATO, 2009, p.97).

Algumas dificuldades inerentes a esse processo podem ser identificadas, dentre as quais podemos citar a seleção adequada de problemas para abordar o conteúdo escolhido e atender aos objetivos propostos, além da alteração no papel do professor, o qual torna-se um orientador do trabalho dos alunos e precisa estar disposto a lidar com imprevistos devido à impossibilidade de prever todos os 
questionamentos, dificuldades e ideias que por eles poderão ser apresentados. No entanto, estas dificuldades não devem ser encaradas como obstáculos, pois a preparação de um plano de aulas, com elementos como os aqui apresentados, que colaborem, por exemplo, para um domínio do conteúdo por parte do professor e para reflexões a respeito de como abordá-lo junto aos alunos, pode auxiliá-lo na superação das dificuldades citadas.

Apesar desta proposta ainda não ter sido por nós aplicada com alunos de Ensino Médio, a expectativa é que os trabalhos a serem desenvolvidos com base na mesma favoreçam a construção de conceitos da Probabilidade, superando a simples memorização de fórmulas, tendo em vista que através da Resolução de Problemas a "formalização dos conceitos e teorias matemáticas, feita pelo professor, passa a fazer mais sentido para os alunos". (ONUCHIC, ALLEVATO, 2011, p. 82).

Com relação à sua utilização no âmbito da formação inicial e continuada de professores de Matemática, destaca-se que pode apresentar potencialidades como auxiliá-los tanto na superação de dificuldades conceituais com o conteúdo matemático quanto a se familiarizarem com essa metodologia de ensino ao vivenciarem no papel de alunos o ensino de Probabilidade através da Resolução de Problemas, o que pode colaborar para que desenvolvam aulas nessa perspectiva, pois conforme destacado por autores como Romanatto $(2008$, p. 5) "existe um aspecto essencial a ser considerado para que professores possam utilizar a resolução de problemas em suas práticas docentes [...], que eles tenham vivenciado em sua formação (inicial e continuada) essa metodologia de ensino".

\section{Referências}

BARBA, A. N. D. Uma Proposta para o Ensino de Probabilidade utilizando a Resolução de Problemas. 2015. 43 f. Monografia (Especialização em Educação Matemática) - Centro de Ciências Exatas, Universidade Estadual de Londrina, Londrina, 2015.

BRASIL. Secretaria de Educação Fundamental. Parâmetros curriculares nacionais: Matemática - terceiro e quarto ciclos. Brasília: MEC/SEF, 1998. 
Ministério da Educação (MEC), Secretaria de Educação Média e Tecnológica (Semtec). PCN + Ensino médio: orientações educacionais complementares aos Parâmetros Curriculares Nacionais - Ciências da Natureza, Matemática e suas Tecnologias. Brasília: MEC/Semtec, 2002.

. Ministério da Educação (MEC). Secretaria de Educação Básica. Orientações Curriculares para o Ensino Médio: Ciências da Natureza, Matemática e suas Tecnologias - Volume 2. Brasília: MEC, 2006.

CAI, J. Helping Elementary School Students Become Successful Mathematical Problem Solvers. In: LAMBDIN, D. V. (Ed.). Teaching and learning mathematics: translating research for elementary school teachers. Reston, VA: NCTM, 2010, p 9-14.

DANTE, L. R. Matemática: contexto e aplicações - Volume 2 - Ensino Médio. São Paulo: Ática, 2013.

IEZZI et. al. Matemática: ciência e aplicações - Volume 2 - Ensino Médio. São Paulo: Saraiva, 2010.

LOPES, J. M. Percepções de professores do ensino médio sobre mudanças de suas práticas de ensino de probabilidade. In: Conferência Interamericana de Educação Matemática, 13, 2011, Recife, Brasil. Disponível em: <http://ciaemredumate.org/ocs/index.php/xiii_ciaem/xiii_ciaem/paper/view/484/118>. Acesso em: 01 dez. 2017.

MAROCCI, L. M.; NACARATO, A. M. Um ambiente de aprendizagem baseado na resolução de problemas: a possibilidade de circulação de significações sobre Probabilidade por meio da linguagem. Educação Matemática Pesquisa, São Paulo, v.15, n.1, p.101-123, 2013.

ONUCHIC, L. R.; ALLEVATO, N. S. G. Trabalhando volume de cilindros através da Resolução de Problemas. Educação Matemática em Revista (Rio Grande do Sul), Canoas, v.1, n.10, p. 95-103, 2009.

ONUCHIC, L. R; ALLEVATO, N. S. G. Pesquisa em Resolução de Problemas: caminhos, avanços e novas perspectivas. Bolema, Rio Claro (SP), v. 25, n. 41, p. 73-98, dez. 2011.

RODRIGUES, V. Resolução de Problemas como estratégia para incentivar e desenvolver a criatividade dos alunos na prática educativa matemática. 1992. Dissertação (Mestrado em Educação Matemática) - Instituto de Geociências e Ciências Exatas, Universidade Estadual Paulista, Rio Claro, 1992.

ROMANATTO, M. C. Resolução de Problemas na Formação de Professores e Pesquisadores. In: Seminário em Resolução de Problemas, 1, 2008. Rio Claro. Anais... Rio Claro, UNESP, 2008. p. 1-9.

ROTUNNO, S. A. M. Estatística e probabilidade: um estudo sobre a inserção desses conteúdos no ensino fundamental. Curitiba, 2007, 117f. Dissertação (Mestrado em Educação) - Universidade Federal do Paraná, Curitiba, 2007. 
http://dx.doi.org/10.5965/2357724X06102018328

SOUZA, J. R. de. Novo Olhar: Matemática - Volume 2. 2.ed. São Paulo: FTD, 2013.

STEIN, M. K.; BOALER; J. SILVER, E. A. Teaching Mathematics through Problem Solving: Research Perspectives. In: SCHOEN, H. L. (Ed.). Teaching Mathematics through Problem Solving: Grades 6-12.Reston, VA: NCTM, 2003. p. 245-256.

TEIXEIRA, B. R.; SANTOS, E. R. Ensino de Matemática através da Resolução de Problemas: alguns aspectos orientadores para a prática docente. Boletim online de Educação Matemática, Joinville, v. 5, n.8, p. 51-71, 2017.

VAN DE WALLE, J. A. Matemática no Ensino Fundamental: formação de professores e aplicação em sala de aula. Porto Alegre: Artmed, 2009. 